\title{
Minimum and maximum against $k$ lies
}

\author{
Michael Hoffmann \\ Institute of Theoretical Computer Science, \\ ETH Zurich, Switzerland \\ E-mail: hoffmann@inf .ethz.ch \\ Jiří Matoušek \\ Department of Applied Mathematics and \\ Institute for Theoretical Computer Science (ITI), \\ Charles University, Prague, Czech Republic \\ E-mail: matousek@kam.mff.cuni.cz \\ Yoshio Okamoto * \\ Graduate School of Information Science and Engineering, \\ Tokyo Institute of Technology, Japan \\ E-mail: okamoto@is.titech.ac.jp \\ Philipp Zumstein \\ Institute of Theoretical Computer Science, \\ ETH Zurich, Switzerland \\ E-mail: zuphilip@inf .ethz.ch
}

November 18, 2018

\begin{abstract}
A neat 1972 result of Pohl asserts that $\lceil 3 n / 2\rceil-2$ comparisons are sufficient, and also necessary in the worst case, for finding both the minimum and the maximum of an $n$-element totally ordered set. The set is accessed via an oracle for pairwise comparisons. More recently, the problem has been studied in the context of the Rényi-Ulam liar games, where the oracle may give up to $k$ false answers. For large $k$, an upper bound due to Aigner shows that $(k+\mathrm{O}(\sqrt{k})) n$ comparisons suffice. We improve on this by providing an algorithm with at most $(k+1+C) n+\mathrm{O}\left(k^{3}\right)$ comparisons for some constant $C$. The known lower bounds are of the form $\left(k+1+c_{k}\right) n-D$, for some constant $D$, where $c_{0}=0.5, c_{1}=\frac{23}{32}=0.71875$, and $c_{k}=\Omega\left(2^{-5 k / 4}\right)$ as $k \rightarrow \infty$.
\end{abstract}

*Supported by Global COE Program "Computationism as a Foundation for the Sciences" and Grant-in-Aid for Scientific Research from Ministry of Education, Science and Culture, Japan, and Japan Society for the Promotion of Science. 


\section{Introduction}

We consider an $n$-element set $X$ with an unknown total ordering $\leq$. The ordering can be accessed via an oracle that, given two elements $x, y \in X$, tells us whether $x<y$ or $x>y$. It is easily seen that the minimum element of $X$ can be found using $n-1$ comparisons. This is optimal in the sense that $n-2$ comparisons are not enough to find the minimum element in the worst case.

One of the nice little surprises in computer science is that if we want to find both the minimum and the maximum, we can do significantly better than finding the minimum and the maximum separately. Pohl $[8$ proved that $\lceil 3 n / 2\rceil-2$ is the optimal number of comparisons for this problem $(n \geq 2)$. The algorithm first partitions the elements of $X$ into pairs and makes a comparison in each pair. The minimum can then be found among the "losers" of these comparisons, while the maximum is found among the "winners."

Here we consider the problem of determining both the minimum and the maximum in the case where the oracle is not completely reliable: it may sometimes give a false answer, but only at most $k$ times during the whole computation, where $k$ is a given parameter.

We refer to this model as computation against $k$ lies. Let us stress that we admit repeating the same query to the oracle several times, and each false answer counts as a lie. This seems to be the most sensible definition-if repeated queries were not allowed, or if the oracle could always give the wrong answer to a particular query, then the minimum cannot be determined.

So, for example, if we repeat a given query $2 k+1$ times, we always get the correct answer by majority vote. Thus, we can simulate any algorithm with a reliable oracle, asking every question $2 k+1$ times, but for the problems considered here, this is not a very efficient way, as we will see.

The problem of finding both the minimum and the maximum against $k$ lies was investigated by Aigner [1], who proved that $(k+\mathrm{O}(\sqrt{k})) n$ comparisons always suffice 1 We improve on this as follows.

Theorem 1. There is an algorithm that finds both the minimum and the maximum among $n$ elements against $k$ lies using at most $(k+1+C) n+\mathrm{O}\left(k^{3}\right)$ comparisons, where $C$ is a constant.

Our proof yields the constant $C$ reasonably small (below 10, say, at least if $k$ is assumed to be sufficiently large), but we do not try to optimize it.

Lower bounds. The best known lower bounds for the number of comparisons necessary to determine both the minimum and the maximum against $k$ lies have the form $\left(k+1+c_{k}\right) n-D$, where $D$ is a small constant and the $c_{k}$ are as follows:

- $c_{0}=0.5$, and this is the best possible. This is the result of Pohl [8] for a truthful oracle mentioned above.

\footnotetext{
${ }^{1}$ Here and in the sequel, $\mathrm{O}($.$) and \Omega($.$) hide only absolute constants, independent of both$ $n$ and $k$.
} 
- $c_{1}=\frac{23}{32}=0.71875$, and this is again tight. This follows from a recent work by Gerbner, Pálvölgyi, Patkós, and Wiener [5] who determined the optimum number of comparisons for $k=1$ up to a small additive constant: it lies between $\left\lceil\frac{87}{32} n\right\rceil-3$ and $\left\lceil\frac{87}{32} n\right\rceil+12$. This proves a conjecture of Aigner [1].

- $c_{k}=\Omega\left(2^{-5 k / 4}\right)$ for all $k$, as was shown by Aigner $[1]$.

The optimal constant $c_{1}=\frac{23}{32}$ indicates that obtaining precise answers for $k>1$ may be difficult.

Related work. The problem of determining the minimum alone against $k$ lies was resolved by Ravikumar, Ganesan, and Lakshmanan [9], who proved that finding the minimum against $k$ lies can be performed by using at most $(k+1) n-1$ comparisons, and this is optimal in the worst case.

The problem considered in this paper belongs to the area of searching problems against lies and, in a wider context, it is an example of "computation in the presence of errors." This field has a rich history and beautiful results. A prototype problem, still far from completely solved, is the Rényi-Ulam liar game from the $1960 \mathrm{~s}$, where one wants to determine an unknown integer $x$ between 1 and $n$, an oracle provides comparisons of $x$ with specified numbers, and it may give at most $k$ false answers. We refer to the surveys by Pelc [7] and by Deppe [2] for more information.

\section{A simple algorithm}

Before proving Theorem 1, we explain a simpler algorithm, which illustrates the main ideas but yields a weaker bound. We begin with formulating a generic algorithm, with some steps left unspecified. Both the simple algorithm in this section and an improved algorithm in the next sections are instances of the generic algorithm.

\section{The generic algorithm}

1. For a suitable integer parameter $s=s(k)$, we arbitrarily partition the considered $n$-element set $X$ into $n / s$ groups $X_{1}, \ldots, X_{n / s}$ of size $s$ each $\theta$

2. In each group $X_{i}$, we find the minimum $m_{i}$ and the maximum $M_{i}$. The method for doing this is left unspecified in the generic algorithm.

3. We find the minimum of $\left\{m_{1}, \ldots, m_{n / s}\right\}$ against $k$ lies, and independently, we find the maximum of $\left\{M_{1}, M_{2}, \ldots, M_{n / s}\right\}$ against $k$ lies.

${ }^{a}$ If $n$ is not divisible by $s$, we can form an extra group smaller than $s$ and treat it separately, say — we will not bore the reader with the details. 
The correctness of the generic algorithm is clear, provided that Step 2 is implemented correctly. Eventually, we set $s:=k$ in the simple and in the improved algorithm. However, we keep $s$ as a separate parameter, because the choice $s:=k$ is in a sense accidental.

In the simple algorithm we implement Step 2 as follows.

\section{Step 2 in the simple algorithm}

21. (Sorting.) We sort the elements of $X_{i}$ by an asymptotically optimal sorting algorithm, say mergesort, using $\mathrm{O}(s \log s)$ comparisons, and ignoring the possibility of lies. Thus, we obtain an ordering $x_{1}, x_{2}, \ldots, x_{s}$ of the elements of $X_{i}$ such that if all queries during the sorting have been answered correctly, then $x_{1}<x_{2}<\cdots<x_{s}$. If there was at least one false answer, we make no assumptions, except that the sorting algorithm does not crash and outputs some ordering.

22. (Verifying the minimum and maximum.) For each $j=2,3, \ldots, s$, we query the oracle $k+1$ times with the pair $x_{j-1}, x_{j}$. If any of these queries returns the answer $x_{j-1}>x_{j}$, we restart: We go back to Step 211 and repeat the computation for the group $X_{i}$ from scratch. Otherwise, if all the answers are $x_{j-1}<x_{j}$, we proceed with the next step.

2.3. We set $m_{i}:=x_{1}$ and $M_{i}:=x_{s}$.

Lemma 1 (Correctness). The simple algorithm always correctly computes the minimum and the maximum against $k$ lies.

Proof. We note that once the processing of the group $X_{i}$ in the above algorithm reaches Step 213, then $m_{i}=x_{1}$ has to be the minimum. Indeed, for every other element $x_{j}, j \geq 2$, the oracle has answered $k+1$ times that $x_{j}>x_{j-1}$, and hence $x_{j}$ cannot be the minimum. Similarly, $M_{i}$ has to be the maximum, and thus the algorithm is always correct.

Actually, at Step 22.3 we can be sure that $x_{1}, \ldots, x_{s}$ is the sorted order of $X_{i}$, but in the improved algorithm in the next section the situation will be more subtle. The next lemma shows, that the simple algorithm already provides an improvement of Aigner's bound of $(k+\mathrm{O}(\sqrt{k})) n$.

Lemma 2 (Complexity). The number of comparisons of the simple algorithm for $s=k$ on an n-element set is $(k+\mathrm{O}(\log k)) n+\mathrm{O}\left(k^{3}\right)$.

Proof. For processing the group $X_{i}$ in Step 2, we need $\mathrm{O}(s \log s)+(k+1)(s-1)=$ $k^{2}+\mathrm{O}(k \log k)$ comparisons, provided that no restart is required. But since restarts may occur only if the the oracle lies at least once, and the total number 
of lies is at most $k$, there are no more than $k$ restarts for all groups together. These restarts may account for at most $k\left(k^{2}+\mathrm{O}(k \log k)\right)=\mathrm{O}\left(k^{3}\right)$ comparisons. Thus, the total number of comparisons in Step 2 is $\frac{n}{s}\left(k^{2}+\mathrm{O}(k \log k)\right)+\mathrm{O}\left(k^{3}\right)=$ $(k+\mathrm{O}(\log k)) n+\mathrm{O}\left(k^{3}\right)$.

As we mentioned in the introduction, the minimum (or maximum) of an $n$-element set against $k$ lies can be found using $(k+1) n-1$ comparisons, and so Step 3 needs no more than $2(k+1)(n / s)=\mathrm{O}(n)$ comparisons. (We do not really need the optimal algorithm for finding the minimum; any $\mathrm{O}((k+1) n)$ algorithm would do.) The claimed bound on the total number of comparisons follows.

\section{The improved algorithm: Proof of Theorem 1}

In order to certify that $x_{1}$ is indeed the minimum of $X_{i}$, we want that for every $x_{j}, j \neq 1$, the oracle declares $x_{j}$ larger than some other element $k+1$ times. (In the simple algorithm, these $k+1$ comparisons were all made with $x_{j-1}$, but any other smaller elements will do.) This in itself requires $(k+1)(s-1)$ queries per group, or $(k+1)(n-n / s)$ in total, which is already close to our target upper bound in Theorem 1 (we note that $s$ has to be at least of order $k$, for otherwise, Step 3 of the generic algorithm would be too costly).

Similarly, every $x_{j}, j \neq s$, should be compared with smaller elements $k+1$ times, which again needs $(k+1)(n-n / s)$ comparisons, so all but $\mathrm{O}(n)$ comparisons in the whole algorithm should better be used for both of these purposes.

In the simple algorithm, the comparisons used for sorting the groups in Step 2,1] are, in this sense, wasted. The remedy is to use most of them also for verifying the minimum and maximum in Step [2]2, For example, if the sorting algorithm has already made comparisons of $x_{17}$ with 23 larger elements, in the verification step it suffices to compare $x_{17}$ with $k+1-23$ larger elements.

One immediate problem with this appears if the sorting algorithm compares $x_{17}$ with some $b>k+1$ larger elements, the extra $b-(k+1)$ comparisons are wasted. However, for us, this will not be an issue, because we will have $s=k$, and thus each element can be compared to at most $k-1$ others (assuming, as we may, that the sorting algorithm does not repeat any comparison).

Another problem is somewhat more subtle. In order to explain it, let us represent the comparisons made in the sorting algorithm by edges of an ordered graph. The vertices are $1,2, \ldots, s$, representing the elements $x_{1}, \ldots, x_{s}$ of $X_{i}$ in sorted order, and the edges correspond to the comparisons made during the sorting, see the figure below on the left.
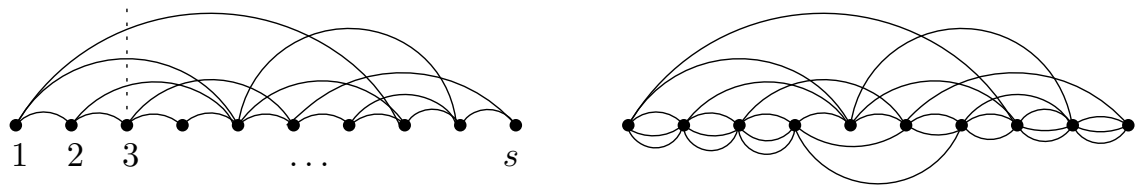
In the verification step, we need to make additional comparisons so that every $x_{j}, j \neq 1$, has at least $k+1$ comparisons with smaller elements and every $x_{j}, j \neq s$, has at least $k+1$ comparisons with larger elements. This corresponds to adding suitable extra edges in the graph, as in the right drawing above (where $k=2$, and the added edges are drawn on the bottom side).

As the picture illustrates, sometimes we cannot avoid comparing some element with more than $k+1$ larger ones or $k+1$ smaller ones (and thus some of the comparisons will be "half-wasted"). For example, no matter how we add the extra edges, the elements $x_{1}, x_{2}, x_{3}$ together must participate in at least 3 half-wasted comparisons. Indeed, $x_{2}$ and $x_{3}$ together require 6 comparisons to the left (i.e. with a smaller element). These comparisons can be "provided" only by $x_{1}$ and $x_{2}$, which together want only 6 comparisons to the right- -but 3 of these comparisons to the right were already made with elements larger than $x_{3}$ (these are the arcs intersecting the dotted vertical line in the picture).

The next lemma shows that this kind of argument is the only source of wasted comparisons. For an ordered multigraph $H$ on the vertex set $\{1,2, \ldots, s\}$ as above, let us define $t(H)$, the thickness of $H$, as $\max \{t(j): j=2,3, \ldots, s-1\}$, where $t(j):=|\{\{a, b\} \in E(H): a<j<b\}|$ is the number of edges going "over" the vertex $j$.

Lemma 3. Let $H$ be an undirected multigraph without loops on $\{1,2, \ldots, s\}$ such that for every vertex $j=1,2, \ldots, s$,

$$
\begin{aligned}
d_{H}^{\text {left }}(j) & :=|\{\{i, j\} \in E(H): i<j\}| \leq k+1, \\
d_{H}^{\text {right }}(j) & :=|\{\{i, j\} \in E(H): i>j\}| \leq k+1 .
\end{aligned}
$$

Then $H$ can be extended to a multigraph $\bar{H}$ by adding edges, so that

(i) every vertex $j \neq 1$ has at least $k+1$ left neighbors and every vertex $j \neq s$ has at least $k+1$ right neighbors; and

(ii) the total number of edges in $\bar{H}$ is at most $(k+1)(s-1)+t(H)$.

The proof is a network flow argument and therefore constructive. We postpone it to the end of this section.

For a comparison-based sorting algorithm $\mathcal{A}$, we define the thickness $t_{\mathcal{A}}(s)$ in the natural way: It is the maximum, over all $s$-element input sequences, of the thickness $t(H)$ of the corresponding ordered graph $H$ (the vertices of $H$ are ordered as in the output of the algorithm and each comparison contributes to an edge between its corresponding vertices). As the above lemma shows, the number of comparisons used for the sorting but not for the verification can be bounded by the thickness of the sorting algorithm.

Lemma 4. There exists a (deterministic) sorting algorithm $\mathcal{A}$ with thickness $t_{\mathcal{A}}(s)=\mathrm{O}(s)$.

Proof. The algorithm is based on Quicksort, but in order to control the thickness, we want to partition the elements into two groups of equal size in each recursive step. 
We thus begin with computing the median of the given elements. This can be done using $\mathrm{O}(s)$ comparisons (see, e.g., Knuth [6]; the current best deterministic algorithm due to Dor and Zwick [3] uses no more than $2.95 s+o(s)$ comparisons). These algorithms also divide the remaining elements into two groups, those smaller than the median and those larger than the median. To obtain a sorting algorithm, we simply recurse on each of these groups.

The thickness of this algorithm obeys the recursion $\left.t_{\mathcal{A}}(s) \leq \mathrm{O}(s)+t_{\mathcal{A}}(\lfloor s / 2\rfloor)\right)$, and thus it is bounded by $\mathrm{O}(s)$.

We are going to use the algorithm $\mathcal{A}$ from the lemma in the setting where some of the answers of the oracle may be wrong. Then the median selection algorithm is not guaranteed to partition the current set into two groups of the same size and it is not sure that the running time does not change. However, we can check if the groups have the right size and if the running time does not increase too much. If some test goes wrong, we restart the computation (similar to the simple algorithm).

Now we can describe the improved algorithm, again by specifying Step 2 of the generic algorithm.

\section{Step 2 in the improved algorithm}

[2.1'. (Sorting.) We sort the elements of $X_{i}$ by the algorithm $\mathcal{A}$ with thickness $\mathrm{O}(s)$ as in Lemma 4. If an inconsistency is detected (as discussed above), we restart the computation for the group $X_{i}$ from scratch.

2, $2^{\prime}$. (Verifying the minimum and maximum.) We create the ordered graph $H$ corresponding to the comparisons made by $\mathcal{A}$, and we extend it to a multigraph $\bar{H}$ according to Lemma 3 . We perform the comparisons corresponding to the added edges. If we encounter an inconsistency, then we restart: We go back to Step $2,1^{\prime}$ and repeat the computation for the group $X_{i}$ from scratch. Otherwise, we proceed with the next step.

2. $3^{\prime}$. We set $m_{i}:=x_{1}$ and $M_{i}:=x_{s}$.

Proof of Theorem 1. The correctness of the improved algorithm follows in the same way as for the simple algorithm. In Step 2l2', the oracle has declared every element $x_{j}, j \neq 1$, larger than some other element $k+1$ times, and so $x_{j}$ cannot be the minimum. A similar argument applies for the maximum.

It remains to bound the number of comparisons. From the discussion above, the number of comparisons is at most $\left((k+1)(s-1)+t_{\mathcal{A}}(s)\right)\left(\frac{n}{s}+k\right)+2(k+1) \frac{n}{s}$, with $t_{\mathcal{A}}(s)=\mathrm{O}(s)$. For $s=k$, we thus get that the number of comparisons at most $(k+1+C) n+\mathrm{O}\left(k^{3}\right)$ for some constant $C$, as claimed. 
Proof of Lemma 3. We will proceed in two steps. First, we construct a multigraph $H^{*}$ from $H$ by adding a maximum number of (multi)edges such that the left and right degree of every vertex are still bounded above by $k+1$. Second, we extend $H^{*}$ to $\bar{H}$ by adding an appropriate number of edges to each vertex so that condition (i) holds.

For an ordered multigraph $H^{\prime}$ on $\{1,2, \ldots, s\}$ with left and right degrees upper bounded by $k+1$, let us define the defect $\Delta\left(H^{\prime}\right)$ as

$$
\Delta\left(H^{\prime}\right):=\sum_{j=1}^{s-1}\left(k+1-d_{H^{\prime}}^{\mathrm{right}}(j)\right)+\sum_{j=2}^{s}\left(k+1-d_{H^{\prime}}^{\mathrm{left}}(j)\right) .
$$

We have $\Delta\left(H^{\prime}\right)=2(k+1)(s-1)-2 e\left(H^{\prime}\right)$, where $e\left(H^{\prime}\right)$ is the number of edges of $H^{\prime}$.

By a network flow argument, we will show that by adding suitable $m^{*}:=$ $(k+1)(s-1)-e(H)-t(H)$ edges to $H$, one can obtain a multigraph $H^{*}$ in which all left and right degrees are still bounded by $k+1$ and such that $\Delta\left(H^{*}\right)=2 t(H)$. The desired graph $\bar{H}$ as in the lemma will then be obtained by adding $\Delta\left(H^{*}\right)$ more edges: For example, for every vertex $j \geq 2$ of $H^{*}$ with $d_{H^{*}}^{\text {left }}(j)<k+1$, we add $k+1-d_{H^{*}}^{\text {left }}(j)$ edges connecting $j$ to 1 , and similarly we fix the deficient right degrees by adding edges going to the vertex $s$.

It remains to construct $H^{*}$ as above. To this end, we define an auxiliary directed graph $G$, where each directed edge $e$ is also assigned an integral capacity $c(e)$; see Figure 1(a).

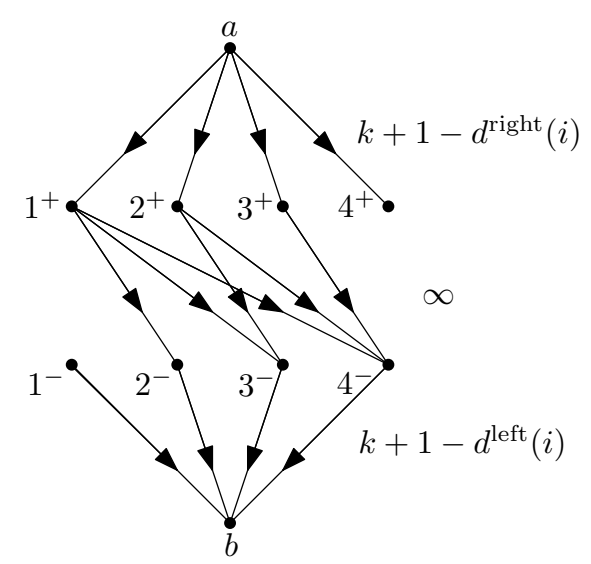

(a) The graph $G$ with capacities.

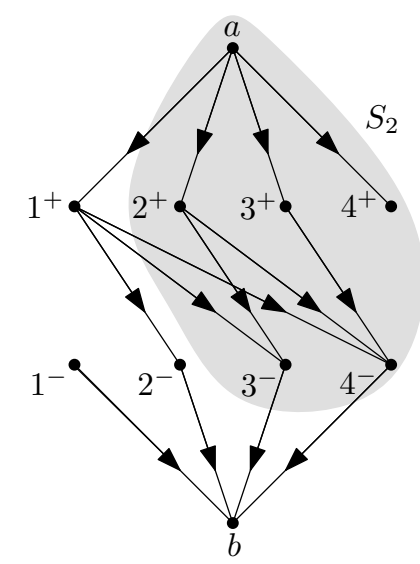

(b) The cut $S_{2}$ in $G$.

Figure 1: The directed graph $G$ constructed in the proof of Lemma 3 ,

The vertex set of $G$ consists of a vertex $j^{-}$for every $j \in\{1,2, \ldots, s\}$, a vertex $j^{+}$for every $j \in\{1,2, \ldots, s\}$, and two special vertices $a$ and $b$. There is a directed edge in $G$ from $a$ to every vertex $j^{+}$and the capacity of this edge is $k+1-d_{H}^{\text {right }}(j)$. Similarly, there is a directed edge in $G$ from every vertex $j^{-}$ 
to $b$, and the capacity of this edge is $k+1-d_{H}^{\text {left }}(j)$. Moreover, for every $i, j$ with $1 \leq i<j \leq s$, we put the directed edge $\left(i^{+}, j^{-}\right)$in $G$, and the capacity of this edge is $\infty$ (i.e., a sufficiently large number).

We will check that there is an integral $a-b$ flow in $G$ with value $m^{*}$ in $G$. By the max-flow min-cut theorem [4], it suffices to show that every $a-b$ cut in $G$ has capacity at least $m^{*}$ and there is an $a-b$ cut in $G$ with capacity $m^{*}$.

Let $S \subseteq V(G)$ be a minimum $a-b$ cut. Let $i$ be the smallest integer such that $i^{+} \in S$. Since the minimum cut cannot use an edge of unbounded capacity, we have $j^{-} \in S$ for all $j>i$.

We may assume without loss of generality that $j^{+} \in S$ for all $j>i$ and $j^{-} \notin$ $S$ for all $j \leq i$ (the capacity of the cut does not decrease by doing otherwise). Therefore it suffices to consider $a-b$ cuts of the form

$$
S_{i}:=\{a\} \cup\left\{x^{+}: x \geq i\right\} \cup\left\{x^{-}: x>i\right\}
$$

for $i=1, \ldots, s$. The capacity of $S_{i}$, see Figure 1(b), equals

$$
\sum_{j<i} c\left(a, j^{+}\right)+\sum_{j>i} c\left(j^{-}, b\right)=(s-1)(k+1)-\sum_{j<i} d_{H}^{\text {right }}(j)-\sum_{j>i} d_{H}^{\text {left }}(j) .
$$

Now let us look at the quantity $\sum_{j<i} d^{\text {right }}(j)+\sum_{j>i} d^{\text {left }}(j)$, and see how much an edge $\left\{j, j^{\prime}\right\}\left(j<j^{\prime}\right)$ of $H$ contributes to it: For $j<i<j^{\prime}$, the contribution is 2 , while all other edges contribute 1 . Hence the capacity of the cut $S_{i}$ is $(k+1)(s-1)-e(H)-t(i)$, and the minimum capacity of an $a$-b-cut is $(k+1)(s-1)-e(H)-t(H)=m^{*}$ as required.

Thus, there is an integral flow $f$ with value $m^{*}$ as announced above. We now select the edges to be added to $H$ as follows: For every directed edge $\left(i^{+}, j^{-}\right)$of $G$, we add $f\left(i^{+}, j^{-}\right)$copies of the edge $\{i, j\}$, which yields the multigraph $H^{*}$. The number of added edges is $m^{*}$, the value of the flow $f$, and the capacity constraints guarantee that all left and right degrees in $H^{*}$ are bounded by $k+1$. Moreover, the defect of $H^{*}$ is at most $2 t(H)$.

\section{Concluding remarks}

We can cast the algorithm when $k=0$ sketched in the introduction into the framework of our generic algorithm. Namely, if we set $s=2$ and in Step 2 we just compare the two elements in each group, then we obtain that algorithm. The main feature of our algorithm is that every restart only spoils one group. This allows us to keep the effect of lies local.

In order to improve the upper bound of Theorem 1 by the method of this paper, we would need a sorting algorithm with thickness $\mathrm{o}(s)$. (Moreover, to make use of the sublinear thickness, we would need to choose $s$ superlinear in $k$, and thus the sorting algorithm would be allowed to compare every element with only o $(s)$ others.) The following proposition shows, however, that such a sorting algorithm does not exist. Thus, we need a different idea to improve Theorem 1 
Proposition 1. Every (randomized) algorithm to sort an s-element set has thickness $\Omega(s)$ in expectation.

Proof. By Yao's principle [10, it is enough to show that every deterministic sorting algorithm $\mathcal{A}$ has expected thickness $\Omega(s)$ for a random input. In our case, we assume that the unknown linear ordering of $X$ is chosen uniformly at random among all the $s$ ! possibilities.

In each step, the algorithm $\mathcal{A}$ compares some two elements $x, y \in X$. Let us say that an element $x \in X$ is virgin at the beginning of some step if it hasn't been involved in any previous comparison, and elements that are not virgin are tainted. A comparison is fresh if it involves at least one virgin element.

For notational convenience, we assume that $s$ is divisible by 8 . Let $L \subset X$ consist of the first $s / 2$ elements in the (random) input order (which is also the order of the output of the algorithm), and let $R:=X \backslash L$. Let $E_{i}$ be the event that the $i$ th fresh comparison is an $L R$-comparison, i.e., a comparison in which one of the two compared elements $x, y$ lies in $L$ and the other in $R$. We claim that for each $i=1,2, \ldots, s / 8$, the probability of $E_{i}$ is at least $\frac{1}{3}$.

To this end, let us fix (arbitrarily) the outcomes of all comparisons made by $\mathcal{A}$ before the $i$ th fresh comparison, which determines the set of tainted elements, and let us also fix the positions of the tainted elements in the input ordering. We now consider the probability of $E_{i}$ conditioned on these choices. The key observation is that the virgin elements in the input ordering are still randomly distributed among the remaining positions (those not occupied by the tainted elements).

Let $\ell$ be the number of virgin elements in $L$ and $r$ the number of virgin elements in $R$; we have $s / 4 \leq \ell, r \leq s / 2$.

We distinguish two cases. First, let only one of the elements $x, y$ compared in the $i$ th fresh comparison be virgin. Say that $x$ is tainted and lies in $L$. Then the probability of $E_{i}$ equals $r /(\ell+r) \geq \frac{1}{3}$.

Second, let both of $x$ and $y$ be virgin. Then the probability of $E_{i}$ is $2 \ell r /((\ell+$ $r)(\ell+r-1))$, and since $s / 4 \leq \ell, r \leq s / 2$, this probability exceeds $\frac{4}{9}$.

Thus, the probability of $E_{i}$ conditioned on every choice of the outcomes of the initial comparisons and positions of the tainted elements is at least $\frac{1}{3}$, and so the probability of $E_{i}$ for a random input is at least $\frac{1}{3}$ as claimed. Thus, the expected number of $L R$-comparisons made by $\mathcal{A}$ is $\Omega(s)$.

Let $a$ be the largest element of $L$, i.e., the $(s / 2)$ th element of $X$, and let $b$ be the smallest element of $R$, i.e., the $(s / 2+1)$ st element of $X$. Since we may assume that $\mathcal{A}$ doesn't repeat any comparison, there is at most one comparison of $a$ with $b$. Every other $L R$-comparison compares elements that have $a$ or $b$ (or both) between them. Thus, the expected thickness of $\mathcal{A}$ is at least half of the expected number of $L R$-comparisons, which is $\Omega(s)$.

Note that the only thing which we needed in the proposition above was that the corresponding ordered graph is simple and has minimum degree at least 1. 


\section{Acknowledgments}

We thank Döm Pálvölgyi for bringing the problem investigated in this paper to our attention. This work has been started at the 7th Gremo Workshop on Open Problems, Hof de Planis, Stels in Switzerland, July 6-10, 2009. We also thank the participants of the workshop for the inspiring atmosphere.

\section{References}

[1] M. Aigner. Finding the maximum and the minimum. Discrete Applied Mathematics 74 (1997) 1-12.

[2] C. Deppe. Coding with feedback and searching with lies. In Entropy, Search, Complexity, Bolyai Society Mathematical Studies 16 (2007), pages $27-70$.

[3] D. Dor and U. Zwick. Selecting the median. SIAM Journal on Computing 28 (1999) 1722-1758.

[4] L. R. Ford Jr. and D. R. Fulkerson. Maximal flow through a network. Canadian Journal of Mathematics 8 (1956) 399-404.

[5] D. Gerbner, D. Pálvölgyi, B. Patkós, and G. Wiener. Finding the maximum and minimum elements with one lie. Submitted.

[6] D. E. Knuth. The Art of Computer Programming. Volume 3. Sorting and Searching. Addison-Wesley Publishing Co. (1973)

[7] A. Pelc. Searching games with errors - fifty years of coping with liars. Theoretical Computer Science 270 (2002) 71-109.

[8] I. Pohl. A sorting problem and its complexity. Communications of the ACM 15 (1972) 462-464.

[9] B. Ravikumar, K. Ganesan, and K. B. Lakshmanan. On selecting the largest element in spite of erroneous information. Proceedings of 4th STACS, Lecture Notes in Computer Science 247 (1987) 88-99.

[10] A. C.-C. Yao. Probabilistic computations: Towards a unified measure of complexity. Proceedings of 18th FOCS (1977) 222-227. 